\title{
Prinzipien der computerassistierten Chirurgie
}

\author{
Richard Bächler, Lutz-Peter Nolte
}

\section{Zusammenfassung}

Die computerassistierte Chirurgie ist ein relativ junges Forschungsgebiet in der Medizin. Chirurgen, Informatiker und Ingenieure haben sich zusammengefunden, um prä- und intraoperative Daten aus bildgebenden Verfahren besser in die Umsetzung der chirurgischen Aktion einzubeziehen. Ziel ist es dabei, die Umsetzung der Planung durch Visualisierung der
Lage und Ausrichtung von Instrumenten in Relation zur operierten Anatomie in Echtzeit zu ermöglichen, um dadurch Eingriffe genauer und sicherer durchführen zu können. Dieser Beitrag erläutert die grundlegenden Bestandteile jedes Systems für die computerassistierte Chirurgie - Anatomie, Bild und Navigator - und beschreibt die in aktuellen Systemen eingesetzten Arten von Bilddaten und Navigatoren.

\section{Einleitung}

Für die Diagnose und Planung von operativen Eingriffen in der orthopädischen Chirurgie steht heutzutage eine ganze Palette von bildgebenden Verfahren zur Verfügung. Dank der laufenden Weiterentwicklung der zur Bilderzeugung eingesetzten Scanner konnten die Genauigkeit und Informationsdichte laufend gesteigert werden, so dass sich praktisch jede Art von Struktur abbilden lässt. Dies führt letztendlich aber zu einer wahren Informationsflut, die der Chirurg mit konventionellen Mitteln kaum noch überblicken kann. So lassen sich z.B. mit einem modernen Spiral-CT in wenigen Sekunden Hunderte einzelner Bilder erzeugen, die sich nur noch mit Computerunterstützung sinnvoll für die Diagnose und präoperative Planung nutzen lassen.

In den letzten Jahren hat sich das Forschungsgebiet der computerassistierten Chirurgie entwickelt, welches Komponenten aus verschiedenen Bereichen, z.B. Bildverarbeitung, bildgebende Verfahren, Robotik, Virtual Reality und Biomechanik einbezieht.

OP-JOURNAL 2002; 17: 4-6

(c) Georg Thieme Verlag Stuttgart . New York
Durch die Verwendung bildgestützter Navigationssysteme, die die präoperative Planung mit der intraoperativen Aktion verbinden, sollen die Sicherheit und Präzision chirurgischer Eingriffe erhöht werden.

Die knochenbasierte orthopädische Chirurgie bietet sich dazu besonders an, da sich das zu operierende Objekt im Gegensatz zu Weichteilen zwischen dem Moment der Bilderzeugung und dem Eingriff kaum verändert. Die vorliegende Arbeit versucht, die grundlegenden Prinzipien der computerassistierten Chirurgie zu erklären und heutzutage eingesetzte Technologien zu beschreiben.

\section{Bestandteile eines Navigationssystems}

Die Funktionsweise eines Navigationssystems und die Klassifikation der verschiedenen Ansätze zur Realisierung lassen sich am einfachsten durch die Betrachtung der Hauptbestandteile verstehen. Die folgenden drei Bestandteile liegen grundsätzlich jedem bildbasierten Navigationssystem zugrunde [1] und werden in der Abb.1 illustriert:

1. Das Therapeutische Objekt bezeichnet den Ort der Behandlung. Als „Behandlung“ wird hier nicht nur ein chirurgischer Eingriff verstanden, sondern auch andere Formen wie z.B. die Bestrahlung in der Radioonkologie.

2. Das Virtuelle Objekt ist ein Abbild des therapeutischen Objekts. Die Art des Abbildes muss im Prinzip nicht eingeschränkt werden, in der Praxis bildbasierter Navigationssysteme sind jedoch hauptsächlich zwei- und dreidimensionale Datensätze von Bedeutung.

3. Der Navigator ist das verbindende Element zwischen therapeutischem und virtuellem Objekt. Er ermöglicht durch das Verfolgen der Anatomie und von chirurgischen Instrumenten die interaktive Darstellung im virtuellen Objekt.

Anhand der neurochirurgischen Stereotaxie lässt sich diese theoretische Beschreibung veranschaulichen: Der Tumor im Schädel, der durch eine Biopsie getroffen werden soll, stellt das therapeutische Objekt dar. Die durch MRI gewonnene Bildinformation dient als virtuelles Objekt, welches die Planung und anschließende Ausführung der Biopsie mit dem Navigator, dem stereotaktischen Rahmen, erlaubt.

Damit ein System für die computerassistierte Chirurgie diese Bestandteile integrieren und damit eine Operationsunterstützung gewährleisten kann, müssen drei wesentliche Vorgänge gelöst werden: die Kalibrierung, die Registrierung und die Referenzierung.

Die Kalibrierung bestimmt die Geometrie der chirurgischen Instrumente in einem dem Navigator bekannten Koordinatensystem. Es handelt sich hierbei um das Definieren wesentlicher Merkmale eines Instruments, z. B. Spitze und Achse eines Bohrers oder Ausrichtung und Form der Schneide eines Meißels. Neben den Instrumenten müssen auch intraoperative bildgebende Systeme kalibriert werden, falls sie mit dem Navigationssystem verwendet werden sollen. Als Beispiel sei hier das Fluoroskop angeführt, bei dem 


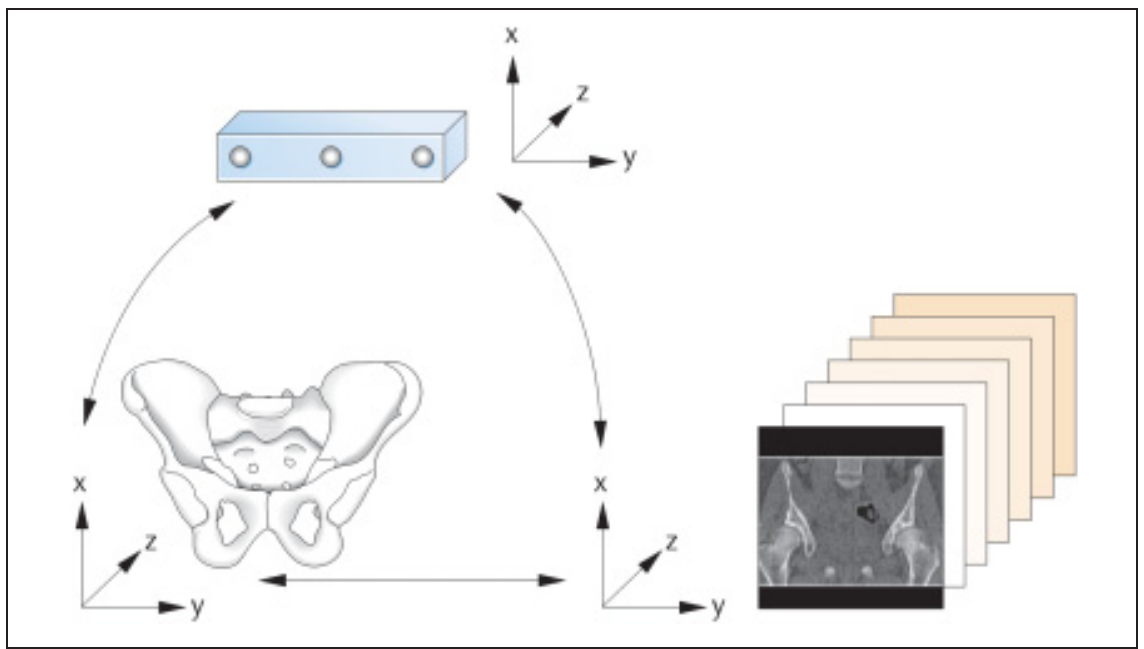

Abb.1 Prinzipdarstellung eines Systems für die computerassistierte Chirurgie: (a) therapeutisches Objekt, (b) virtuelles Objekt, (c) Navigator.

zusätzlich zur Lage des Bildwandlers auch die Bestimmung der Projektionsparameter notwendig ist.

Um die Lage der Instrumente in Bezug auf das therapeutische Objekt präzis im virtuellen Objekt wiedergeben zu können, muss eine Relation zwischen den Koordinatensystemen der Objekte hergestellt werden. Dieser Vorgang wird Registrierung genannt und erlaubt es, jeden Punkt im Raum des einen Objekts im Raum des anderen Objekts abzubilden. Im Gegensatz zur Kalibrierung muss die Registrierung für jeden Eingriff neu ausgeführt werden. Je nach dem verwendeten Registrierungsalgorithmus müssen im Raum des therapeutischen und virtuellen Objekts unterschiedliche Strukturen definiert werden. Im einfachsten Fall sind dies Punktepaare, die auf anatomischen oder künstlichen Landmarken basieren, und die vom Chirurg sowohl im therapeutischen wie im virtuellen Objekt leicht bestimmt werden können. Im intraoperativen Situs geschieht dies meist mit einem speziellen Digitalisierpointer. Etwas aufwändigere Algorithmen verwenden neben Punkten auch Kurven und Oberflächen der Objekte als Eingabedaten, um Mehrdeutigkeitsprobleme während der Berechnung auszuschließen.

Da die Registrierung nur schwer automatisierbar ist und von einer hohen Genauigkeit der Eingabedaten abhängt, stellt sie die Hauptfehlerquelle bei der computerassistierten Chirurgie dar.

Der Chirurg muss deshalb durch eine sorgfältige Verifizierung überprüfen, dass das Navigationssystem die Lage der chirurgischen Instrumente auch korrekt darstellt. Lavallée [2] gibt einen guten Überblick über die verwendeten Techniken und mögliche Probleme.

Um minimalinvasive Eingriffe zu ermöglichen, befasst sich die aktuelle Forschung mit dem berührungslosen Bestimmen der für die Registrierung notwendigen Strukturen. Dazu werden im Operationssaal bereits vorhandene Geräte beigezogen, wie z.B. Ultraschallsonden oder Fluoroskopiesysteme, oder neue Verfahren entwickelt, wie z.B. laserbasierte Oberflächenscanner. Gleichzeitig sollen auch der für die Registrierung notwendige Aufwand verringert und die Wahrscheinlichkeit von Bedienerfehlern minimiert werden.

Da sich die Lage des therapeutischen Objekts in Bezug auf den Navigator während eines chirurgischen Eingriffes ändern kann, muss dieser Einfluss kompensiert werden. Dies geschieht durch die statische bzw. die dynamische Referenzierung. Bei der statischen Referenzierung wird zwischen dem therapeutischen Objekt und dem Navigator eine feste Verbindung hergestellt, z.B. durch eine Knochenklemme oder Verschraubung. Bei einer Vielzahl von Eingriffen in der Orthopädie ist diese Technik jedoch nicht praktikabel, da der Zugang zum Situs eingeschränkt würde. Aus diesem Grund wird die so genannte dynamische Referenzierung verwendet, die es dem Navigator in Echtzeit erlaubt, relative Bewegungen des therapeutischen Objekts zu erfassen und zu kompensieren. Dazu wird mittels einer Klemme oder Schraube am zu operierenden Knochen ein Sensor befestigt, der dem Navigator als Bezugspunkt zum therapeutischen Objekt dient.

\section{Klassifizierung}

Eine Klassifizierung von Systemen für die computerassistierte Chirurgie anhand des therapeutischen Objekts ist zwar möglich, die wesentlichen Unterschiede verschiedener Systeme ergeben sich aber aus der Betrachtung der Art des virtuellen Objektes und des verwendeten Navigators.

Die der Planung und nachfolgenden intraoperativen Navigation zugrunde liegende Repräsentation des virtuellen Objekts richtet sich nach den Bedürfnissen der chirurgischen Aktion.

Die ersten Mitte der 90er Jahre in der Orthopädie eingesetzten Navigationssysteme für die Pedikelschraubeninsertion basierten auf einem dreidimensionalen CT-Datensatz. Dieser erlaubt eine exakte Planung der späteren Schraubenlage ebenso wie das Definieren von anatomischen Strukturen, die der späteren Registrierung dienen. CT- und auch MRI-Datensätze finden heute in der Orthopädie, vor allem im Beckenbereich bei komplexen dreidimensionalen Aufgaben, ihre Anwendung.

Mit der Integration eines intraoperativ verfügbaren Fluoroskops in ein Navigationssystem entfällt die Notwendigkeit einer präoperativen CT-Studie. Durch das Aufnehmen von zweidimensionalen Röntgenbildern aus verschiedenen Winkeln lässt sich eine pseudodreidimensionale Darstellung erzielen. Da die Lage der Instrumente in mehreren Ansichten in Echtzeit angezeigt werden kann, müssen nur einige wenige Aufnahmen geschossen werden, was die Strahlenbelastung im Vergleich zur konventionellen Fluoroskopie im Dauerbetrieb erheblich reduziert. Neueste Entwicklungen im Bereich der Fluoroskopie erlauben sogar die Erzeugung eines echten dreidimensionalen Datensatzes mit einer dem CT vergleichbaren Qualität. Bei der fluoroskopiebasierten Navigation reduziert sich zudem das Problem der Registrierung auf eine Kalibrierung, was einen Vorteil gegenüber der CT- oder MRI-basierten Navigation darstellt.

Eine weitere Möglichkeit, das therapeutische Objekt abzubilden, stellt die so genannte „chirurgendefinierte“ Anatomie dar. Bei dieser Darstellungsform, 
die heute unter anderem bei Anwendungen am Kniegelenk eingesetzt wird, tastet der Chirurg die für die Ausführung der Operation notwendigen anatomischen Strukturen direkt im Situs ab. Werden z.B. beim vorderen Kreuzbandersatz die vorgesehenen Ansatzpunkte des neuen Bandes und die Oberflächen von Tibia und Femur digitalisiert, lässt sich die Belastung des Implantats und ein allfälliges Impingement vor dem Einsetzen am Monitor visualisieren.

Ein weiteres Mittel zur Klassifikation von Navigationssystemen stellt der verwendete Navigator dar. Grundsätzlich können drei verschiedene Arten von Navigatoren unterschieden werden, deren Eigenschaften kurz vorgestellt werden sollen. Es handelt sich dabei um passive, aktive und semiaktive Navigatoren.

Passive Navigatoren erlauben es dem Chirurg, seine Instrumente frei und weitestgehend unbehindert zu führen. Durch eine spezifische Adaptation der Instrumente an das Navigationssystem können Lage und Orientierung im Raum in Echtzeit relativ zum therapeutischen Objekt gemessen werden. Die heute am weitesten verbreiteten passiven Navigatoren sind optoelektronische Kamerasysteme, die an den Instrumenten angebrachte Leuchtdioden oder reflektierende Kugeln verfolgen. Als Mittel zur Positionsbestimmung dienen Infrarotlichtimpulse, die bei den Leuchtdioden aktiv abgestrahlt werden (sog. aktive Marker) und bei den Kugeln zur Kamera reflektiert werden (sog. passive Marker). Die Verwendung von passiven Markern erlaubt die Gestaltung von Instrumenten ohne Kabel und bei geringem zusätzlichen Gewicht. Dem steht die höhere Genauigkeit und Verlässlichkeit der aktiven Marker gegenüber. Passive Navigatoren auf der Basis von Vielgelenkarmen, akustischen oder magnetischen Sensoren konnten sich für den Einsatz im Operationssaal bis jetzt nicht durchsetzen. Bei den Gelenkarmen beeinträchtigt die stark eingeschränkte Bewegungsfreiheit und die Problematik der statischen Referenzierung die Verwendung. Akustische und magnetische Systeme reagieren sehr sensibel auf Veränderungen in ihrer Umwelt, was die Genauigkeit und Verlässlichkeit im Vergleich zu den optoelektronischen Systemen stark beeinträchtigt.

Aktive Navigatoren, auch als Operationsroboter bezeichnet, führen eine chirurgi- sche Aktion selbständig durch. Der ROBODOC als Pioniersystem in der Orthopädie kann so die Femurschafthöhlung gemäß einer präoperativen Planung mit sehr hoher Präzision ausfräsen. Dies führt zu einer höheren Primärstabilität bei unzementierten Schäften. Zusätzlich erlaubt das System, bei Revisionsoperationen einen allenfalls vorhandenen Zementmantel exakt zu entfernen, eine Aufgabe, die sich manuell nur schwer erledigen lässt. Die Geschichte des ROBODOC zeigt auf, dass die Entwicklung eines Operationsroboters sehr anspruchsvoll ist, um den hohen Sicherheitsanforderungen im Operationssaal - keine Gefährdung des Patienten und des Personals - gerecht werden zu können. Die Verwendung von aktiven Navigatoren ist nicht nur auf die Orthopädie beschränkt. So werden auch in anderen Gebieten der Chirurgie Robotersysteme für die Operationsunterstützung entwickelt. Viele dieser Systeme befinden sich jedoch noch im Laborstadium oder in der klinischen Erprobung.

Die letzte Gruppe von Navigatoren, die semiaktiven Navigatoren, stellen eine Mischform aus aktiven und passiven Systemen dar. Die eigentliche chirurgische Aktion wird zwar vom Arzt ausgeführt, der Navigator beschränkt dabei aber die Bewegungsfreiheit auf Bereiche, die in der präoperativen Planung als sicher gekennzeichnet wurden. Der einfachste mechanische semiaktive Navigator, der routinemäßig verwendet wird, ist der stereotaktische Rahmen. Hier kann die am virtuellen Objekt präoperativ geplante Trajektorie intraoperativ am Rahmen eingestellt werden, so dass anschließend eine Biopsienadel entsprechend der Planung kontrolliert eingeführt werden kann. Neueste Forschungsarbeiten beschäftigen sich damit, mikrochirurgische Eingriffe mit Hilfe semiaktiver Navigatoren zu unterstützen. Bei diesen Anwendungen werden einerseits durch den Arzt verursachte Schwingungen des Instruments ausgeblendet, andererseits kann das System als Übersetzungsgetriebe fungieren, das großräumige Bewegungen des Arztes verkleinert und so Aktionen im Submillimeterbereich erlaubt. Eine große Herausforderung stellt in diesem Zusammenhang die Integration von haptischem Feedback dar, auf das sich der Chirurg während der Ausführung eines Eingriffs verlässt.

\section{Schlussfolgerungen}

Systeme für die computerassistierte Chirurgie haben sich im Bereich der Orthopädie, der Neurochirurgie und HNO etabliert und sind in einer steten Verbreitung begriffen. Den größten Anteil stellen dabei Systeme auf der Basis von passiven, optoelektronischen Navigatoren. Da die Sicherheitsanforderungen an aktive und semiaktive Navigationssysteme erheblich höher liegen, haben bis jetzt nur zwei Systeme in der Orthopädie kommerziellen Status erreicht. Trotzdem darf in diesem Gebiet in den kommenden Jahren einiges erwartet werden, ist doch in der Literatur schon eine Reihe von viel versprechenden Ansätzen beschrieben worden.

Die Bedeutung der computerassistierten Chirurgie wird in den nächsten Jahren noch zunehmen. Die vielfältigen Möglichkeiten der präoperativen Planung und intraoperativen Navigation haben ihr Potenzial zur Erhöhung der Sicherheit und Präzision bei der Ausführung von chirurgischen Eingriffen bewiesen. Dank der Forschung auf diesem Gebiet wird es auch weiterhin Verbesserungen und Weiterentwicklungen geben. Diese werden z.B. der Erschließung von neuen Anwendungsbereichen dienen, aber auch Einfluss auf den Bereich der Simulation von Operationen nehmen, was einen Einsatz für die Aus- und Weiterbildung von Chirurgen ermöglicht.

\section{Literatur}

1 Bowersox JC, Bucholz RD, Delp SL et al. Excerpts from the final report for the Second International Workshop on Robotics and Computer Assisted Medical Interventions, June 23, 1996, Bristol, England. Comput Aided Surg 1997; 2: 69-101

2 Lavallée S. Registration for computer-integrated surgery: methodology, state of the art. In: Taylor RH, Lavallée S, Burdea GC Mösges R (eds.): Computer-integrated surgery. MIT Press, Cambridge London 1996; $77-97$

\section{Dr. phil.-nat. Richard Bächler Gruppenleiter \\ Prof. Dr.-Ing. Lutz-Peter Nolte \\ Abteilungsleiter}

M. E. Müller-Institut für Biomechanik Universität Bern

Murtenstrasse 35

CH-3010 Bern 